\title{
Philosophiques
}

\section{Commentaire sur L’inquiétude de la pensée}

\section{Claudia Serban}

Volume 44, numéro 2, automne 2017

URI : https://id.erudit.org/iderudit/1042341ar

DOI : https://doi.org/10.7202/1042341ar

Aller au sommaire du numéro

Éditeur(s)

Société de philosophie du Québec

ISSN

0316-2923 (imprimé)

1492-1391 (numérique)

Découvrir la revue

Citer ce document

Serban, C. (2017). Commentaire sur L'inquiétude de la pensée. Philosophiques, 44(2), 357-363. https://doi.org/10.7202/1042341ar d'utilisation que vous pouvez consulter en ligne.

https://apropos.erudit.org/fr/usagers/politique-dutilisation/ 


\title{
Commentaire sur L'inquiétude de la pensée
}

\author{
CLAUDIA SERBAN
}

Université Toulouse-Jean-Jaurès

«La vie et non l'être, tel est le motif initial de la pensée du jeune Heidegger », insiste Sophie-Jan Arrien dans son Précis. L'objectif de son livre a été en effet de remonter dans le parcours philosophique de Heidegger en deçà de la question de l'être, pour faire apparaître une interrogation qui est non seulement tout aussi fondamentale, mais aussi irréductible à celle-ci. Ainsi, malgré la synthèse que le titre du cours fribourgeois de 1923, Ontologie: Herméneutique de la facticité laisse trop facilement supposer comme allant de soi, l'auteure a montré, comme son Précis le souligne encore avec force, que "l'herméneutique phénoménologique de la vie facticielle» ne saurait se laisser réduire "à une proto-ontologie, même "fondamentale" ". Cette mise au point ouvre la possibilité de conférer aux premiers cours fribourgeois de Heidegger le statut d'un corpus autonome tout en jetant sur l'analytique existentiale un soupçon sans doute ancien, mais justifié autrement et nouvellement, à savoir celui d'une impossibilité foncière, sinon d'un refus de penser la vie. L'on peut en effet mettre en opposition et en tension ces deux affirmations que moins d'une décennie sépare: ce passage du cours de 1919-1920 sur les Problèmes fondamentaux de la phénoménologie, que Sophie-Jan Arrien met en avant dans son Précis: "Ontologie - le mot même révèle déjà que le problème décisif [de la philosophie] n'est pas aperçu: histoire et vie» (GA 58, p. 146), et la phrase célèbre d'Être et Temps selon laquelle "La vie est un mode d'être spécifique (eine eigene Seinsart), mais il n'est essentiellement accessible que dans le Dasein (nur zugänglich im Dasein)" (Sein und Zeit, p. 59). Cette dernière assertion contient malgré elle l'aveu d'une aporie: si la vie est un mode d'être spécifique, pourquoi ne serait-elle accessible que dans le Dasein? Qu'est-ce qui barre ici l'accès direct à la vie? Pourquoi l'approche philosophique de la vie devrait-elle prendre le chemin détourné de l'ontologie (fondamentale) et consister simplement en une interprétation privative de l'existence?

L'herméneutique de la vie facticielle du jeune Heidegger, dont L'inquiétude de la pensée restitue les ambitions et les enjeux, permet au contraire de découvrir une phénoménologie (in extremis ontologique) de la vie qui s'édifie par une description et une interprétation de manifestations de la vie auxquelles l'accès ne saurait être supposé médiat ou détourné. Ce qui ne veut pas dire qu'avec l'accessibilité directe des phénomènes de la vie, toute question méthodique devienne superflue: au contraire, pour Heidegger, comme le rappelle encore le Précis de l'auteure, «tout le défi de son herméneutique de la vie, à titre de science originaire, sera de ne pas immobiliser la vie facticielle (ou facticité) dans des concepts intemporellement valides et 
dans des contenus de sens idéaux pris comme normes du savoir. Elle devra au contraire maintenir vive la mutabilité ou mobilité (Bewegtheit) constitutive de la vie sans chercher à en apaiser l'inquiétude (Unrube) native, qui n'est autre que l'inquiétude de la pensée elle-même — d'où le titre de l'ouvrage ».

Dans cette caractérisation de l'entreprise du jeune Heidegger, deux points attirent notre attention: d'une part, le souci de la «mobilité constitutive de la vie " et la nécessité "préserver au sein même de ses concepts l'événement vécu du sens qui l'a vue naître " (nous reviendrons, dans ce qui suit, sur cette conjonction remarquable entre mobilité et événementialité dont Sophie-Jan Arrien nous invite à mesurer la portée); et d'autre part, l'identification de la mobilité de la vie à l'inquiétude de la pensée. Pour ce qui en est du dernier point, il faut concéder qu'il ne va pas si facilement de soi: comment accepter ce paradoxe qui veut que l'attention à la vie se confonde avec l'acte de la philosophie? Pourtant, nous rappelle encore le Précis, dans l'herméneutique phénoménologique qu'élabore le jeune Heidegger, «vie et philosophie, objet et méthode, se trouvent [...] liés l'un à l'autre de façon indissoluble, au point même de se confondre ». L'enjeu est alors de taille: il s'agit de doter cette apparente résorption de la vie dans l'activité philosophique d'une signification positive, non réductionniste, en opérant un double mouvement de transfiguration conceptuelle, pour assurer à la fois l'ancrage ou la descente de la philosophie dans la vie, et l'accession ou l'ascension de la vie à la philosophie. Le ressort de ce remaniement qui confère au logos de la vie une "double acception" (logos de la vie et vie du logos) n'est autre que le "caractère auto-interprétatif de la vie », autrement dit, le fait que le sens est l'élément même de la vie.

Le Précis de Sophie-Jan Arrien, tout comme l'ouvrage dont il reprend les résultats, insiste sur ce statut nodal de la question de la philosophie: l'accomplissement de la vie facticielle, ou son acte propre, tourne autour de la pratique philosophique, là où l'existence résolue du Dasein, telle que la dépeint l'analytique de 1927 , n'est pas à comprendre d'abord comme existence philosophique. Ou, s'il faut encore durcir le trait: "le Dasein n'est philosophe, si l'on veut, que de façon accidentelle». Et si l'on songeait à objecter que ce n'est là, pour le Heidegger d'Être et Temps, qu'un moyen d'achever définitivement la rupture avec le primat traditionnel de la theoria, il faudrait alors rappeler que l'activité philosophique en question a renoncé depuis longtemps à ses anciens habits théoriques. L'une des forces remarquables de l'herméneutique de la vie facticielle du jeune Heidegger réside alors, nous le comprenons, dans sa radicale non-indifférence à la question de la philosophie, ressaisie comme praxis; ou mieux encore, dans son engagement dans la philosophie qui est simultanément engagement dans l'existence et dans l'histoire. "La philosophie n'est pas indifférente à la vie, elle est pour la vie une manière de se retrouver elle-même et de s'enrichir; il s'agit si l'on veut d'une philosophie engagée ", lit-on encore dans le Précis de l'auteure. Or nous pouvons soupçonner que c'est précisément cette cohésion 
originaire de l'engagement dans la philosophie, dans l'existence et dans l'histoire que la pensée ultérieure de Heidegger n'a pas su maintenir. Une dislocation et une fracture interne ont affecté par la suite, sans doute dès l'opus magnum de 1927, à la fois le sens de la vie et le sens de la philosophie.

Mais d'où venait leur unité originelle? La constellation conceptuelle qui permet le mieux de la ressaisir est sans doute celle que forment la mobilité (Bewegtheit) de la vie, l'événementialité (Ereignischarakter) du vécu et l'accomplissement (Vollzug) de la situation vécue. L'herméneutique de la vie facticielle repose en effet, comme le rappelle Sophie-Jan Arrien dans son Précis, sur "la saisie vécue du phénomène en tant que "performance" signifiante, en tant qu'événement appropriant et approprié à travers ce vécu ". Or c'est précisément cette "performance signifiante " qui sauve les concepts philosophiques d'une immobilité rigide et de l'aridité. Le jeune Heidegger en prend progressivement la mesure grâce à certaines confrontations décisives que les analyses de L'inquiétude de la pensée ont fait ressortir dans toute leur portée. Si Aristote, saint Paul et saint Augustin, mais aussi Luther et Kierkegaard, seront pour Heidegger des compagnons privilégiés dans son travail d'exégèse du sens de la vie, il est aussi montré que le projet philosophique d'Emil Lask a contribué de manière décisive à révéler à l'élève de Heinrich Rickert «l'exigence de sortir des perspectives strictement logique et épistémologique» (op. cit., p. 59) dont Husserl était encore resté largement tributaire. La tâche nouvelle et élargie de la philosophie prendra d'abord chez Heidegger, comme le montre le chapitre II de l'ouvrage, intitulé " $\mathrm{Du}$ vécu à la vie", la forme d'un "travail descriptif sur le vécu» (op. cit., p. 70) qui s'intéresse d'abord à sa donation. Sophie-Jan Arrien insiste également sur la nouvelle compréhension du vécu comme événement (Ereignis) - voire, comme événement appropriant (p. 73) - qui se trouve au cœur de cette refonte de la phénoménologie. En effet, le cadre d'analyse de l'herméneutique de la vie facticielle, fût-il de part en part différent de celui des thématisations ultérieures de l'Ereignis, invite dans une égale mesure à insister sur la dimension d'appropriation qui se joue dans le caractère événementiel du vécu: comme le souligne l'auteure, «le vécu m’arrive et, m'arrivant, me fait proprement arriver à moi-même à chaque fois, me fait m'approprier» (p. 74). S'il peut donc être légitime de se référer à un "événement appropriant de 1919» (p. 73), cette façon de parler est tout de même contrainte de se résigner devant le fait que «Heidegger ne précise toutefois pas explicitement la nature de cet événement appropriant duquel se reçoit le monde du soi dans la mesure où il abandonne le concept sitôt après l'avoir découvert» (p. 74).

À ce propos, nous souhaiterions émettre une hypothèse et la soumettre à l'auteure sous forme de question. Si le caractère d'événement du vécu reste, avec sa qualification comme Ereignis en 1919, dans une certaine mesure encore vague, ne peut-on pas considérer qu'il se précisera plus tard (dès le semestre d'hiver 1919-1920) par la mise en avant de la dimension 
d'effectuation ou d'accomplissement (Vollzug) de l'expérience vécue? Heidegger écrit en effet dans son cours de 1919-1920 intitulé Problèmes fondamentaux de la phénoménologie: «L'être de la vie, des vécus ne signifie pas: se produire (Vorkommen), mais: accomplissement. Accomplissement dans le soi, sans que le soi soit toujours et nécessairement présent de manière expresse » (GA 58,156). Ce passage autorise, nous semble-t-il, à mettre en parallèle les deux couples d'opposés présents dans les premiers cours fribourgeois: événement - processus (GA 56/57, $\mathbb{S} 15)$ et accomplissement - fait d'arriver ou de se produire (GA 58,156). On peut dès lors estimer que le recours à la notion de Vollzug vise précisément à décrire le caractère indissolublement événementiel et appropriant des vécus, ou ce qui les distingue radicalement de la manière dont arrive un processus naturel ou psychologique.

Si le fait que l'événement est susceptible d'être rapproché ici de l'accomplissement peut paraître paradoxal, il s'explique pourtant à condition d'entendre l'événement comme événement appropriant: l'accomplissement propre au vécu comme événement n'est autre que l'appropriation. C'est pourquoi il n'est à nos yeux en aucun cas prématuré ou hasardeux de parler, comme le fait Sophie-Jan Arrien, d'un «événement appropriant de 1919» (antérieur donc, nous le comprenons, à l'Ereignis présent dans la pensée de Heidegger dans les années 30 ). Et si cette première occurrence bien précoce de l'Ereignis reste plutôt fulgurante et ne connaîtra pas de vraie postérité ou de pérennité au sein de l'herméneutique de la facticité du jeune Heidegger, il nous semble que c'est la mise en avant de l'accomplissement qui prend très tôt la relève de l'événementialité des vécus, et elle ne peut le faire que pour autant que cette événementialité doit effectivement être comprise comme appropriation.

Une fois qu'on a rendu justice au caractère événementiel du vécu, la question sur laquelle se concentrent les analyses de L'inquiétude de la pensée est de nature méthodologique: "Au sein de quelle science peut être sauvegardé le caractère d'Er-eignis, étranger à toute objectivation, de l'expérience vécue?» (p. 83). C'est pour répondre à cette question que l'auteure examine la voie méthodologique ouverte par l'intuitionnisme husserlien, ainsi que la mise en cause de cette voie sous la plume de Natorp. Si, d'une part, Heidegger ne peut que souscrire au refus de Natorp d' "accréditer le "principe des principes" " (p. 90), d'autre part, il ne saurait accepter « la médiation théorique que ce dernier avance en guise de solution", qui "ne fait que repousser d'un cran le problème de la donation originaire des vécus ». Ce qui apparaît ainsi, c'est «la faiblesse de la reconstruction» natorpienne (p. 98), qui se réduit, en dernière instance, à une démarche d'objectivation et de théorisation. Le jeune Heidegger devra dès lors frayer la voie de sa phénoménologie herméneutique de la vie en renvoyant dos à dos Husserl et Natorp.

Quelle sera en conséquence la résolution apportée au problème méthodologique d'une science préthéorique de la vie? Elle exige d'abord, comme 
le rappelle l'auteure, de "s'attaque[r] simultanément au problème de l'intuition et à celui de l'expression ", jusqu'à en dégager cette synthèse inédite que sera l'intuition herméneutique (p. 103). Mais cette tâche requiert aussi une révision radicale de la conceptualité philosophique, pour faire apparaître la possibilité de concepts qui «ne relèvent ni de la généralisation, ni de la formalisation» (p. 113), des concepts que Sophie-Jan Arrien propose de rayer (typographiquement), afin de laisser ressortir tout l'écart qui les sépare de la conceptualité traditionnelle de la philosophie. Cette nécessité de «biffer» ou de «rayer» les concepts philosophiques traditionnels répond directement à l'exigence de "préserver leur mouvement inquiet » et de respecter "l'événementialité du sens» (p. 207 et 358). Plus loin encore, la spécificité de l'acte herméneutique opéré sur une vie elle-même compréhensive permet de préserver la dimension préthéorique du vécu au sein de la description: comme le souligne l'auteure, "la vie facticielle ne se débarrasse pas, grâce à l'interprétation explicative, de la compréhension originaire en vue d'un comprendre "objectif", mais éclaire ce comprendre primaire» (p. 194). C'est à cette condition seulement que le logos de la philosophie sera à même d'amplifier et de faire résonner sans contradiction et sans écart objectivant le logos de la vie.

Si le traitement des défis de méthode qui se sont présentés à l'entreprise du jeune Heideggeer a montré que "seule l'indication formelle permet de saisir le véritable point de départ de la destruction" (p. 212), il faudra ensuite (et c'est ce que feront les analyses du chapitre IV de L'inquiétude de la pensée) se livrer à la reconstitution successive de la destruction des concepts de vie, d'histoire et de vécu. Après cette première étape de la démarche destructive, le chapitre V poursuivra «La destruction de l'expérience facticielle de la vie ", en réactivant pour commencer l'évidence de départ selon laquelle «le problème des formes authentiques du philosopher apparaît conjointement à la question des structures originaires de la vie » (p. 266). C'est en vertu de cette coappartenance du problème de la vie et du problème de la philosophie que le versant positif de la destruction herméneutique exigera l'" "explosion" des catégories traditionnelles» en vue de découvrir «les articulations mobiles de la vie et de son être-là facticiel» (p. 268). La destruction, nous le comprenons, est par excellence l'outil méthodique permettant d'infuser la vie dans la philosophie, et la philosophie dans la vie.

Enfin, l'insistance sur le fait que "Heidegger s'est d'abord tourné vers Aristote (et vers l'être) pour répondre à la question non pas de l'être mais de la vie» (p. 318) est, comme nous l'avons déjà relevé, à même d'apporter un amendement capital des diagnostics exégétiques — entérinés le plus souvent par l'auto-interprétation rétrospective du philosophe de Messkirch — selon lesquels la voie de la philosophie se confondrait d'entrée de jeu, dans le parcours de Heidegger, avec la voie de l'ontologie. Dans le cadre de l'herméneutique de la facticité, le jeune professeur fribourgeois s'intéresse en effet à un 
Aristote plus physique, éthique et psychologique que logique et métaphysique. C'est notamment la manière qu'a Heidegger de «renvo[yer] la facticité au problème (aristotélicien) de la kinesis» (p. 330), ou la "réorientation "cinétique" de la facticité », qui sont soulignées par Sophie-Jan Arrien, pour affirmer que «l'association de la facticité comme sens d'être de la vie à l'idée du mouvement, de la mobilité en tant que telle, est ce qui manifeste le plus clairement l'irruption d'Aristote dans la pensée de la vie du jeune Heidegger » (p. 337). Mais Aristote lui permettra également de «formaliser» la description des manifestations de la vie facticielle conquise préalablement à l'aide de saint Paul et de saint Augustin. Cette formalisation - ou "appropriation "dé-théologisée" de ces auteurs chrétiens " (p. 356) — contribuera à entraîner la destitution du paradigme historique considéré initialement comme étant «le plus profond» en vue de penser la facticité, à savoir le (proto-)christianisme, en invitant à «troquer la perspective d'une vie bienheureuse en Dieu contre l'expérience authentique de la philosophie » (p. 319), ou plus précisément encore, en échangeant une manière de philosopher contre une autre: philosopher avec Dieu ou sans Dieu. Sophie-Jan Arrien montre aussi que c'est par une interprétation de la phronèsis aristotélicienne que Heidegger réussit à avancer vers une définition et une compréhension pré- ou non-théorique de la philosophie "comme agir, comme effectuation concrète de la vie en vue d'elle-même, et donc comme praxis", dans un "retournement complet» (p. 354) du primat, posé par le Stagirite, de la theoria, de la contemplation qui permet de s'immortaliser et de se rapprocher du divin.

C'est également comme un effet de la formalisation entamée sous l'égide d'Aristote que peut se comprendre, dans le discours heideggérien, l'apparition d'une "ontologie qui s'égalera en 1923 à une herméneutique de la facticité " (p. 324), là où, de prime abord, la réflexion sur le "sens d'être du "je suis" tend[ait] même à sortir de tout horizon ontologique» (p. 242). À travers le prisme de l'équivalence tacite entre ontologie et herméneutique de la facticité que pose le titre du cours de l'été 1923, il appert en effet que "le passage de la vie à l'être ne se fait pas, chez le jeune Heidegger, sous le mode de l'exclusion ni même de la substitution ", dans la mesure où «la question de l'être n'apparaît qu'en lien avec l'exploration du phénomène de la vie» (p. 324). L'infléchissement ontologique du questionnement est donc requis avant tout par le «logos de la vie» (p. 339), même s'il contribuera par la suite à miner les conditions d'entente de ce dernier. Faire résonner à l'unisson le logos de la facticité et le logos de la philosophie, et ce faisant, répondre au défi de "doter le philosopher du logos qui lui soit adéquat" (p. 351), voici la prouesse inégalée de l'herméneutique de la facticité du jeune Heidegger.

En revanche - et par là la conclusion de l'ouvrage anticipe sur le Précis de l'auteure - , dans le passage de l'herméneutique de la vie facticielle à l'ontologie fondamentale, "c'est tout un pan de cette mobilité de la pensée engagée dans le monde qui disparaîtra au regard» (p. 359). Un diagnostic 
critique peut ainsi être formulé, à partir des acquis de l'herméneutique de la facticité, non seulement à l'égard d'Être et Temps, mais aussi, sans doute, à propos de toute la philosophie ultérieure de Heidegger, sous la forme du reproche d'une fragilisation ou d'une exténuation de l'" unité en expansion toujours concrète, dynamique et différenciée de la vie» (p. 360). L'ouvrage de Sophie-Jan Arrien n'a ainsi pas tort de rappeler, à sa dernière page, "la grandeur et le danger qui guettent le penser quand il se maintient dans l'inquiétude de la pensée véritable» (p. 361). 